\title{
Design of Evolutionally Optimized Rule-Based Fuzzy Neural Networks Based on Fuzzy Relation and Evolutionary Optimization
}

\author{
Byoung-Jun Park ${ }^{1}$, Sung-Kwun $\mathrm{Oh}^{2}$, Witold Pedrycz ${ }^{3}$, and Hyun-Ki Kim ${ }^{2}$ \\ ${ }^{1}$ Department of Electrical Electronic and Information Engineering, Wonkwang University, \\ 344-2, Shinyong-Dong, Iksan, Chon-Buk, 570-749, South Korea \\ ${ }^{2}$ Department of Electrical Engineering, The University of Suwon, San 2-2 Wau-ri, \\ Bongdam-eup, Hwaseong-si, Gyeonggi-do, 445-743, South Korea \\ ohsk@suwon.ac.kr \\ ${ }^{3}$ Department of Electrical and Computer Engineering, University of Alberta, Edmonton, \\ AB T6G 2G6, Canada and Systems Research Institute, Polish Academy of Sciences, \\ Warsaw, Poland
}

\begin{abstract}
In this paper, new architectures and comprehensive design methodologies of Genetic Algorithms (GAs) based Evolutionally optimized Rule-based Fuzzy Neural Networks (EoRFNN) are introduced and the dynamic searchbased GAs is introduced to lead to rapidly optimal convergence over a limited region or a boundary condition. The proposed EoRFNN is based on the Rulebased Fuzzy Neural Networks (RFNN) with the extended structure of fuzzy rules being formed within the networks. In the consequence part of the fuzzy rules, three different forms of the regression polynomials such as constant, linear and modified quadratic are taken into consideration. The structure and parameters of the EoRFNN are optimized by the dynamic search-based GAs.
\end{abstract}

\section{Introduction}

In this paper, new architectures and comprehensive design methodologies of Genetic Algorithms [1] (GAs) based Evolutionally optimized Rule-based Fuzzy Neural Networks (EoRFNN) are introduced for effective analysis and solution of nonlinear problem and complex systems. The proposed EoRFNN is based on the Rule-based Fuzzy Neural Networks (RFNN). In the consequence part of the fuzzy rules, three different forms of the regression polynomials such as constant, linear and modified quadratic are taken into consideration. The polynomial of a fuzzy rule results from that we look for a fuzzy subspace (a fuzzy rule) influencing the better output of a model, and then raise the order of polynomial of the fuzzy rule (subspace). Contrary to the former, we make a simplified form for the representation of a fuzzy subspace lowering of the performance of a model. This methodology can effectively reduce the number of parameters and improve the performance of a model. GAs being a global optimization technique determines optimal parameters in a vast search space. But it cannot effectively avoid a large amount of time-consuming iteration because GAs finds optimal parameters by using a given space (region). To alleviate the problems, the dynamic search-based GAs is introduced to lead to rapidly optimal convergence over a limited 
region or a boundary condition. To assess the performance of the proposed model, we exploit a well-known numerical example [2], [3].

\section{Polynomial Fuzzy Inference Architecture of Rule Based FNN}

The networks are classified into the two main categories according to the type of fuzzy inference, namely, the simplified and linear fuzzy inference. In this paper, we propose the polynomial fuzzy inference based RFNN (pRFNN). The proposed pRFNNs are obtained from the integration and extension of conventional RFNNs.

[Layer 1] Input layer

[Layer 2] Computing activation degrees of linguistic labels

[Layer 3] Computing firing strength of premise rules

[Layer 4] Normalization of a degree activation (firing) of the rule

[Layer 5] Multiplying a normalized activation degree of the rule by connection weight [Layer 6] Computing output of pRFNN

The proposed pRFNN can be designed to adapt a characteristic of a given system, also, that has the faculty of making a simple structure out of a complicated model for a nonlinear system, because the pRFNN comprises consequence structure with various orders (Types) for fuzzy rules.

\section{Evolutionally Optimized Rule-Based Fuzzy Neural Networks}

In order to generate the proposed EoRFNN, the dynamic search based GAs is used in the optimization problems of structures and parameters. From the point of fuzzy rules, these divide into the structure and parameters of the premise part, and that of consequence part. The structure issues in the premise of fuzzy rules deal with how to use of input variables (space) influencing outputs of model. The selection of input variables and the division of space are closely related to generation of fuzzy rules that determine the structure of RFNN, and govern the performance of a model. Moreover, a number of input variable and a number of space divisions induce some fatal problems such as the increase of the number of fuzzy rules and the time required. Therefore, the relevant selection of input variables and the appropriate division of space are required. The structure of the consequence part of fuzzy rules is related to how represents a fuzzy subspace. Universally, the conventional methods offer uniform types to each subspace. However, it forms a complicated structure and debases the output quality of a model, because it does not consider the correlation of input variables and reflect a feature of fuzzy subspace. In this study, we apply the various forms in expressions of a fuzzy subspace. The form is selected according to an influence of a fuzzy subspace for an output criterion and provides users with the necessary information of a subspace for a system analysis.

\section{Experimental Studies}

In this experiment, we use three-input nonlinear function as in [2], [3]. This dataset was analyzed using Sugeno's method [2]. We consider 40 pairs of the original input- 
output data. 20 out of 40 pairs of input-output data are used as learning set and the remaining part serves as a testing set.

Fig. 1 shows topologies of EoRFNN. In order to solve a given nonlinear problem, these architectures are designed and generated in flexibility that can cope with an environment (condition).

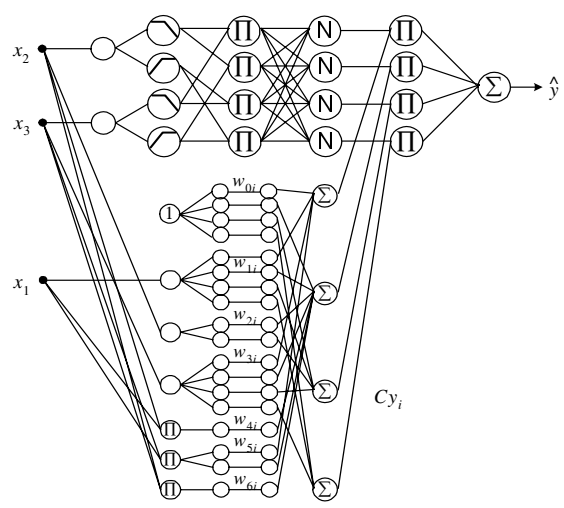

Fig. 1. Topology of EoRFNN02 with 2 inputs for the nonlinear function

Table 1 covers a comparative analysis including several previous models. Sugeno's model I and II were fuzzy models based on linear fuzzy inference method while Shinichi's models formed fuzzy rules by using learning method of neural networks. The study of literature [5] is based on fuzzy-neural networks using HCM clustering and evolutionary fuzzy granulation. Multi-FNN consists of 3 FNN structures. The proposed EoRFNNs come with higher accuracy and improved prediction capabilities.

Table 1. Comparison of performance with other modeling methods

\begin{tabular}{|c|c|c|c|c|}
\hline \multicolumn{2}{|c|}{ Model } & $\mathrm{PI}$ & E_PI & No. of rules \\
\hline \multicolumn{2}{|c|}{ Linear model [3] } & 12.7 & 11.1 & \\
\hline \multicolumn{2}{|c|}{ GMDH $[3,6]$} & 4.7 & 5.7 & \\
\hline \multirow{3}{*}{ Sugeno's $[2,3]$} & Fuzzy I & 1.5 & 2.1 & 3 \\
\hline & Fuzzy II & 1.1 & 3.6 & 4 \\
\hline & FNN Type 1 & 0.84 & 1.22 & $8\left(2^{3}\right)$ \\
\hline \multirow[t]{2}{*}{ Shinichi's [4] } & FNN Type 2 & 0.73 & 1.28 & $4\left(2^{2}\right)$ \\
\hline & FNN Type 3 & 0.63 & 1.25 & $8\left(2^{3}\right)$ \\
\hline \multirow{2}{*}{ FNN [5] } & Simplified & 2.865 & 3.206 & $9(3+3+3)$ \\
\hline & Linear & 2.670 & 3.063 & $9(3+3+3)$ \\
\hline \multirow{2}{*}{ Multi-FNN [5] } & Simplified & 0.865 & 0.956 & $9(3+3+3)$ \\
\hline & Linear & 0.174 & 0.689 & $9(3+3+3)$ \\
\hline \multirow{2}{*}{\multicolumn{2}{|c|}{$\begin{array}{l}\text { Proposed model } \\
\text { (EoRFNN) }\end{array}$}} & 0.241 & 0.357 & $8(2 \times 2 \times 2)$ \\
\hline & & 0.224 & 0.643 & $4(2 \times 2)$ \\
\hline
\end{tabular}




\section{Concluding Remarks}

In this paper, new architectures and comprehensive design methodologies of Evolutionally optimized Rule-based Fuzzy Neural Networks (EoRFNN) has discussed for effective analysis and solution of nonlinear problem and complex systems. Also, the dynamic search-based GAs has introduced to lead to rapidly optimal convergence over a limited region or a boundary condition. The proposed EoRFNN is based on the Rule-based Fuzzy Neural Networks (RFNN) with the extended structure of the fuzzy rules. The structure and parameters of the proposed EoRFNN are optimized by GAs. The proposed EoRFNN can be designed to adapt a characteristic of a given system, also, that has the faculty of making a simple structure out of a complicated model for a nonlinear system. This methodology can effectively reduce the number of parameters and improve the performance of a model. The proposed EoRFNN can be efficiently carried out both at the structural as well as parametric level for overall optimization by utilizing the separate (A) or consecutive (B) tuning technology. From the results, (B) methodologies simultaneously tuning both structure and parameters, reduce parameters of consequence part, and offer the output performance better than the (A). Namely, (B) method is effective in identifying a model than (A).

Acknowledgements. This work has been supported by KESRI(I-2004-0-074-0-00), which is funded by MOCIE(Ministry of commerce, industry and energy)

\section{References}

1. Goldberg, D.E.: Genetic Algorithms in search, Optimization \& Machine Learning. Addison-wesley. (1989)

2. Kang, G., Sugeno, M.: Fuzzy Modeling. Transactions of the Society of Instrument and Control Engineers. 23(6) (1987) 106-108

3. Park, M.Y. and Choi, H.S.: Fuzzy Control System. Daeyoungsa. (1990) 143-158 (In Korean)

4. Horikawa, S.I., Furuhashi, T., Uchigawa, Y.: On Fuzzy Modeling Using Fuzzy Neural Networks with the Back Propagation Algorithm. IEEE Transactions on Neural Networks. 3(5) (1992) 801-806

5. Park, H.S., Oh, S.K.: Multi-FNN Identification Based on HCM Clustering and Evolutionary Fuzzy Granulation. International Journal of Control, Automation and Systems. 1(2) (2003) 194-202

6. Kondo, T.: Revised GMDH algorithm estimating degree of the complete polynomial. Transactions of the Society of Instrument and Control Engineers. 22(9) (1986) 928-934

7. Park, H.S., Oh, S.K.: Fuzzy Relation-based Fuzzy Neural-Networks Using a Hybrid Identification Algorithm. International journal of Control, Automations, and Systems. 1(3) (2003) 289-300

8. Park, H.S., Oh, S.K.: Rule-based Fuzzy-Neural Networks Using the Identification Algorithm of the GA Hybrid Scheme. International Journal of Control, Automations, and Systems. 1(1) (2003) 101-110 